\title{
Analysis of laser acceleration in a semi-infinite space as inverse transition radiation
}

\author{
T. Plettner \\ Stanford Linear Accelerator Center, Stanford, CA 94025
}

\begin{abstract}
This article calculates the energy gain of a single relativistic electron interacting with a single gaussian beam that is terminated by a metallic reflector at normal incidence by two different methods: the electric field integral along the path of the electron, and the overlap integral of the transition radiation pattern from the conductive foil with the laser beam. It is shown that for this instance the two calculation methods yield the same expression for the expected energy change of the electron.
\end{abstract}

\section{INTRODUCTION}

A recent proof-of-principle experiment for laser-driven particle acceleration [1] employed a single linearly polarized laser beam interacting with a $30 \mathrm{MeV}$ relativistic electron beam in a semi-infinite vacuum terminated by a thin metallic boundary. The high reflective surface prevents the laser beam from interacting with the electron beam in the space downstream of the boundary. Figure 1 illustrates the accelerator setup used in the proof-of-principle experiment, which is similar to the arrangement originally proposed by Edinghofer and Pantell [2].

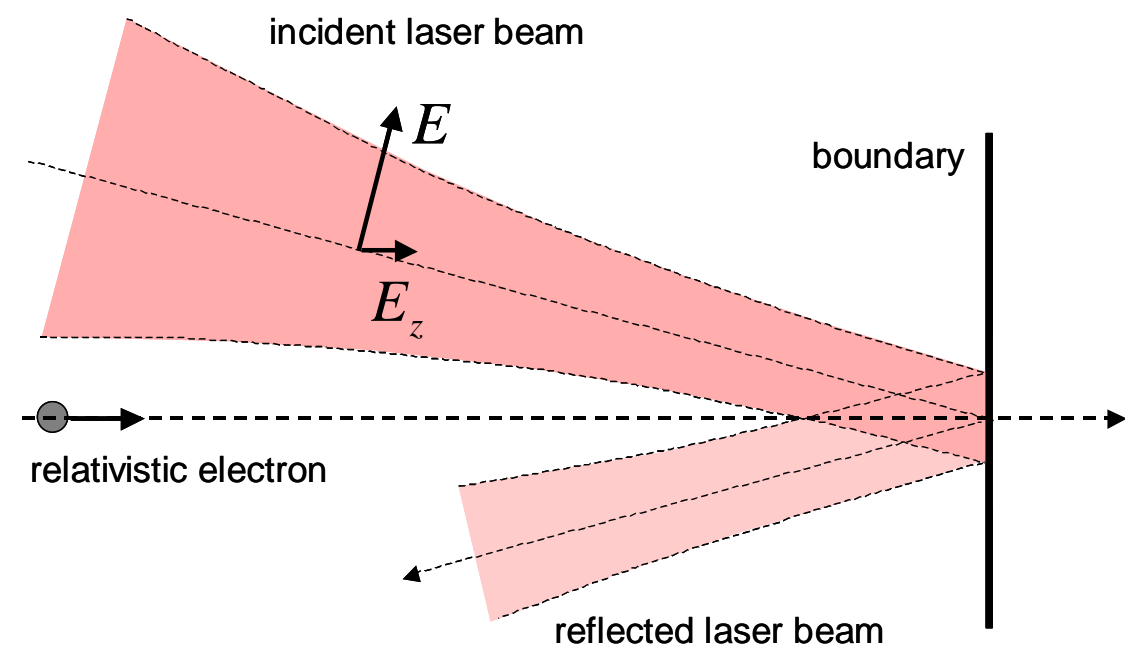

FIG 1: The laser beam, electron beam and boundary configuration.

$E$ is the electric field of the laser and $E_{z}$ is the field component parallel to the electron beam trajectory. For this simple setup the energy gain for the electron beam is most conveniently calculated by integrating the electric field component of laser beam that is 
parallel to the electron beam $\left(E_{z}\right)$ along its trajectory over the interaction length with the laser. As shown in Figure 1 this corresponds to the semi-infinite space upstream of the boundary. Assuming that the external field causes no significant change in the particle's trajectory the energy gain caused by the external laser field is

$$
\Delta U=\int_{a}^{b} q \vec{E}(\vec{r}, t) d \vec{r}
$$

The energy gain observed in the proof-of-principle experiment was in good agreement with the energy gain expected by employing Equation 1. It was observed to scale linearly with the amplitude of the laser electric field, showed the expected laser polarization dependence and occurred only in the presence of the field-terminating boundary.

Many other laser-driven particle acceleration schemes employ Equation 1 to calculate the energy gain. Examples include crossed gaussian laser beams [3], Hermite-gaussian laser beams [4], and other arbitrary laser beam or light diffraction electric field profiles [5]. In addition to semi-open free space laser accelerators other schemes like Inverse-Cerenkov accelerators [6] and guided-wave accelerators [7] (optical or RF) also employ the path integral method of Equation 1 to estimate the electron beam energy gain.

Equation 1 results from integrating the Lorentz force acting on the electron over its path where it interacts with the external field. However, Poynting's Theorem offers an alternative method for calculating the energy change of the electron. Poynting's Theorem [8] states that inside a given volume $V$

$$
\Delta U=-\Delta W_{E M}-\int_{-\infty}^{\infty} \oint_{S} \frac{1}{\mu_{0}}\left(\vec{E}_{\text {total }} \times \vec{B}_{\text {total }}\right) \cdot \hat{n} d s d t
$$

where $\Delta U$ is the mechanical work on the electric charges in the volume $V, \Delta W_{E M}$ is the change of the electromagnetic energy stored in the volume $V$, and the last term is the energy flux leaving the volume $V$ through its boundary $S . \vec{E}_{\text {total }}$ and $\vec{B}_{\text {total }}$ are the total electric and magnetic fields at the boundary $S$.

It has been shown that for the case of an electron interacting with an external electromagnetic field (like a laser) Equation 2 can be rewritten to express the energy change of the electron as the overlap integral of the wake field of the electron in the presence of the structure or medium with the laser field $[9,10]$.

$$
\Delta U=-\int_{-\infty}^{\infty} \oint_{S} \frac{2}{Z_{0}}\left(\vec{E}_{\text {laser }} \cdot \vec{E}_{\text {wake }}\right) d s d t
$$

where $\Delta U$ now represents the energy gain of the charged particle. The quantities $\vec{E}_{\text {laser }}$ and $\vec{E}_{\text {wake }}$ are the laser and electron fields in the presence of the structure [10]. 
Figure 2 illustrates a generalized situation of an electron interacting with a structure or medium in combination with an external laser field.

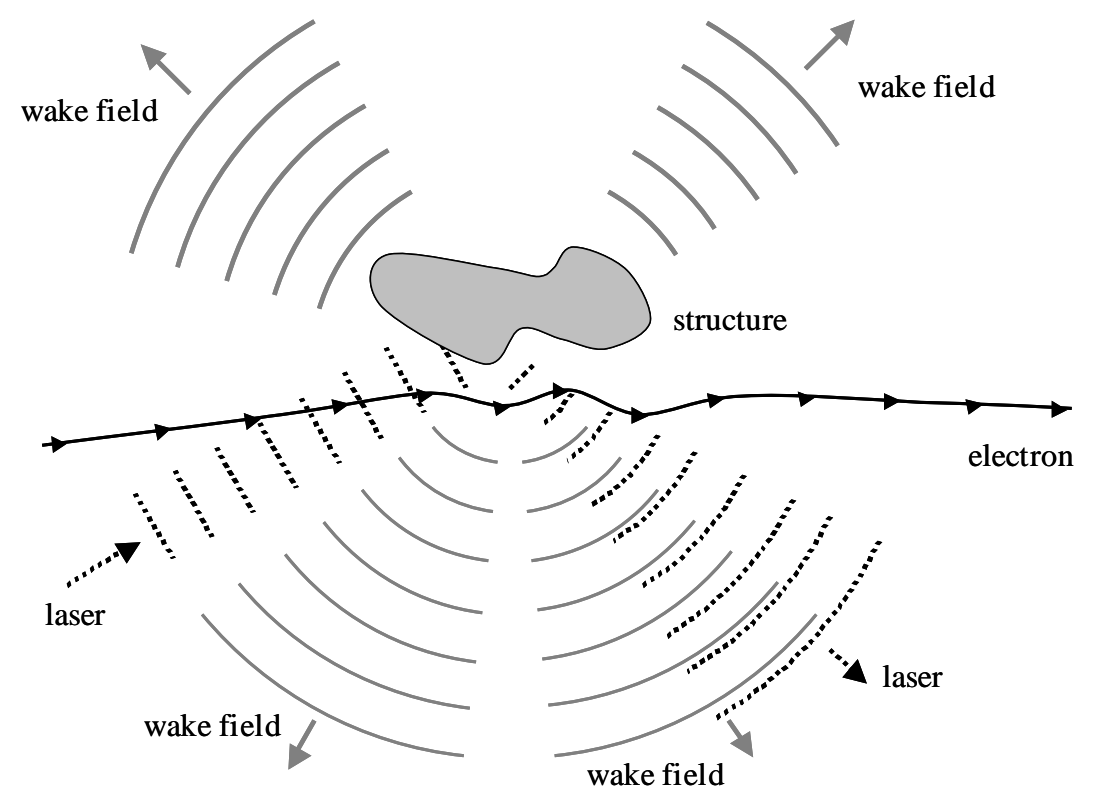

FIG 2: schematic of overlap integral of laser and wake field radiation

The overlap integral Equation expresses laser acceleration as an inverse-radiation process. However, several important assumptions are made in Equation 3 and are important to keep in mind when making generalizations:

- The wake field amplitude is much smaller than the external driving amplitude, that is $\left|\vec{E}_{\text {wake }}\right|<<\left|\vec{E}_{\text {laser }}\right|$. Thus one can neglect the wake field radiation contribution from the accelerator structure or medium. This can be Cerenkov, aperture, transition radiation or even undulator radiation in a wiggler, or any combination of these depending on the specific structure.

- The orbit of the electron suffers no appreciable change in the presence of the driving field. For relativistic electrons this is usually a valid approximation.

- There is no change in the stored electromagnetic energy in the volume where the acceleration is calculated, thus $\Delta W_{E M}=0$.

- There is no ohmic loss in the accelerator structure or medium, such that the energy flux of the laser into the volume is equal to the energy flux of the laser exiting the volume, and furthermore the only contribution to $\Delta U$ comes from the energy gain of the electron beam and not from currents in the medium.

- Other possible non-radiative scattering losses are neglected

These assumptions are realistic for most laser-acceleration experiments, such as the laseracceleration proof-of-principle LEAP experiment, and hence it is of interest to calculate the energy change of the electron beam by the inverse-radiation picture described in Equation 3 and compare it to the familiar path-integral energy gain calculation method. 


\section{THE PATH INTEGRAL ENERGY GAIN METHOD}

A linearly polarized $\mathrm{TEM}_{00}$ gaussian laser beam is assumed to interact with a relativistic electron beam in free space over a finite distance. The expression for the gaussian beam electric field magnitude is derived by the assumption that the laser beam profile variation in the transverse dimension is much smaller than the variation due to the optical phase in the longitudinal dimension. With these assumptions, and derived as a scalar, the amplitude of the transverse electric field component is [11]

$$
E_{\text {transverse }}\left(x^{\prime}, y^{\prime}, z^{\prime}, t\right)=\frac{E_{0} e^{-\frac{x^{\prime 2}+y^{\prime 2}}{w\left(z^{\prime}\right)^{2}}}}{\sqrt{1+z^{\prime 2} / z_{0}^{2}}} \cos \left(\omega t-k z^{\prime}-\eta\left(z^{\prime}\right)-\frac{k x^{\prime 2}}{2 R\left(z^{\prime}\right)}-\varphi\right) \quad 4
$$

The coordinates $\left(x^{\prime}, y^{\prime}, z^{\prime}\right)$ are aligned to the laser beam, where $\left(x^{\prime}, y^{\prime}\right)$ are the transverse coordinates while $z^{\prime}$ is the direction of propagation of the laser beam. $E_{0}$ is the peak electric field amplitude of the laser beam, $\varphi$ is a phase offset angle and $\omega$ is the frequency of the laser. $z_{0}$ is the Raleigh range given by $z_{0}=\pi w_{0}{ }^{2} / \lambda w\left(z^{\prime}\right)$, where $w_{0}$ is the beam waist and $\lambda$ is the wavelength. $w\left(z^{\prime}\right)=w_{0}\left(1+\left(\lambda z^{\prime}\right)^{2} /\left(\pi w_{0}\right)^{2}\right)$ is the beam size located at $z^{\prime}, \quad R\left(z^{\prime}\right)=z\left(1+z_{0}^{2} / z^{\prime 2}\right)$ is the radius of curvature at $z^{\prime}$, and $\eta\left(z^{\prime}\right)=\tan ^{-1}\left(z^{\prime} / z_{0}\right)$ is the Guoy phase shift for a TEM $\mathrm{TE}_{00}$ gaussian laser beam at $z^{\prime}$. The focus of the beam in Equation 1 occurs at $z^{\prime}=0$. As shown in Figure 3 the coordinates $\left(x^{\prime}, y^{\prime}, z^{\prime}\right)$ are rotated by an angle $\alpha$ about the y-axis with respect to a coordinate system $(x, y, z)$ aligned with the electron beam.

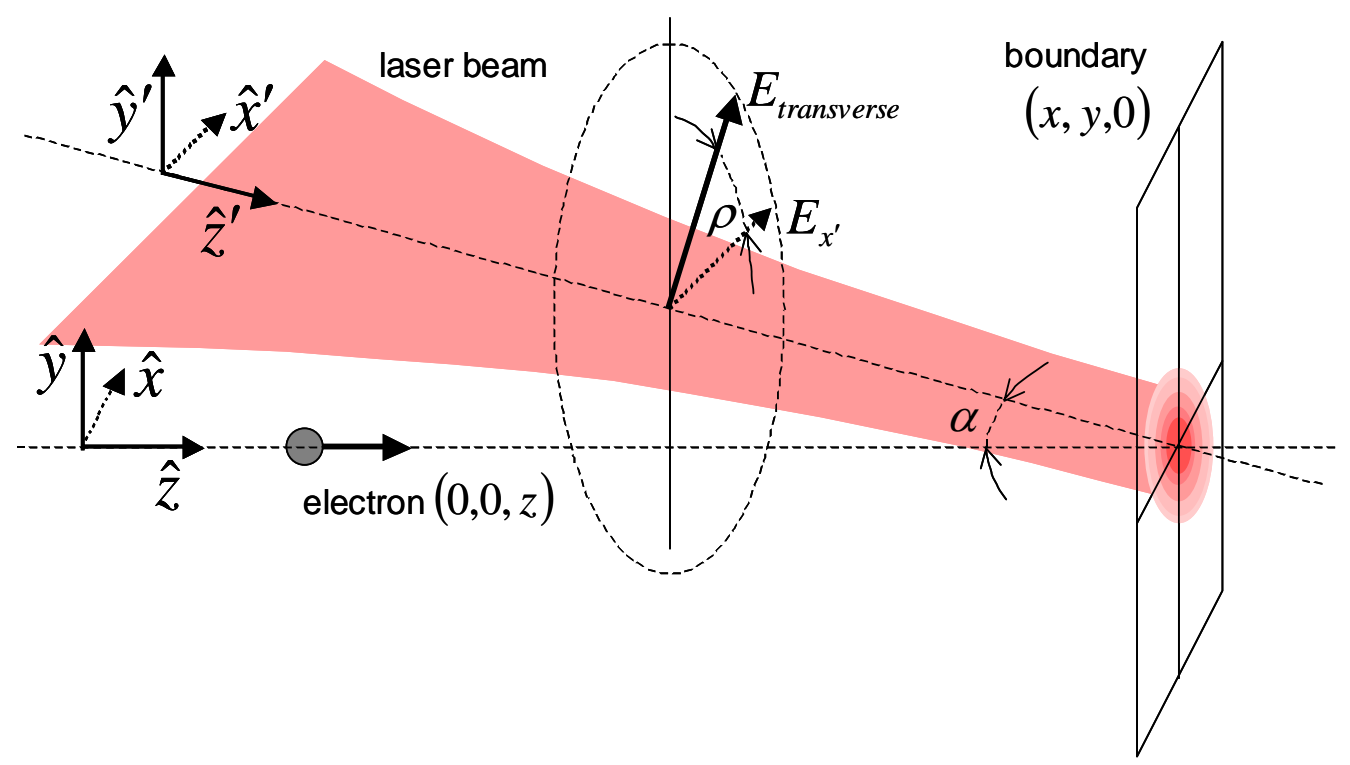

FIG 3: Schematic diagram of the laser beam and the electron beam incident on a thin boundary 
Figure 3 illustrates a linearly polarized laser beam oriented at an angle $\alpha$ with respect to the electron beam and at a polarization angle $\rho$. The electron travels along the coordinates $(0,0, z(t))$ where $z(t)=c \beta t$. To calculate the energy gain of the particle the field component $E_{z}(0,0, z(t), t)$ has to be evaluated and integrated along the z-axis.

Since $E_{\text {transverse }}$ is a scalar quantity the orientation of this field in the $(x, y, z)$ coordinates has to be added by hand. Allowing for the polarization angle $\rho$ the orientation vector for the transverse electric field is

$$
\hat{n}_{\text {laser }}=\left(\begin{array}{c}
\cos \alpha \cos \rho \\
\sin \rho \\
\sin \alpha \cos \rho
\end{array}\right)
$$

Thus the expression for the $\mathrm{TEM}_{00}$ gaussian laser beam in the $(x, y, z)$ coordinates is

$$
\vec{E}(x, y, z, t)=\frac{E_{0} e^{-\frac{x^{\prime 2}+y^{\prime 2}}{w\left(z^{\prime}\right)^{2}}}}{\sqrt{1+z^{\prime 2} / z_{0}^{2}}} \cos \left(\omega t-k z^{\prime}-\eta\left(z^{\prime}\right)-\frac{k x^{\prime 2}}{2 R\left(z^{\prime}\right)}-\varphi\right)\left(\begin{array}{c}
\cos \alpha \cos \rho \\
\sin \rho \\
\sin \alpha \cos \rho
\end{array}\right)
$$

With this expression for the laser electric field one can perform the path integral energy estimate in the spirit as shown in Equation 1. The electric field component parallel to the direction of propagation of the electron beam and along the electron beam axis is

$$
E_{z}(0,0, z, t)=\frac{E_{0} e^{-\frac{x^{\prime 2}+y^{\prime 2}}{w\left(z^{\prime}\right)^{2}}}}{\sqrt{1+z^{\prime 2} / z_{0}^{2}}} \cos \left(\omega t-k z^{\prime}+\eta\left(z^{\prime}\right)+\frac{k x^{\prime 2}}{2 R\left(z^{\prime}\right)}-\varphi\right) \sin \alpha \cos \rho
$$

where the $\left(x^{\prime}, y^{\prime}, z^{\prime}\right)$ coordinates correspond to $x^{\prime}=z \sin \alpha, y^{\prime}=0, z^{\prime}=z \cos \alpha$. With these coordinate substitutions Equation 7 simplifies to

$$
\begin{aligned}
& E_{z}=\frac{E_{0} \sin \alpha \cos \rho}{\left(1+\hat{z} \cos ^{2} \alpha\right)^{1 / 2}} e^{-\left(\frac{\hat{z} \cdot \sin \alpha / \theta_{d}}{1+\hat{z}^{2}}\right)} \cos \psi_{t} \\
& \psi_{t}=\omega t-k z \cos \alpha+\eta(z \cos \alpha)+\frac{k z^{2} \sin ^{2} \alpha}{2 R(z \cos \alpha)}-\varphi
\end{aligned}
$$

The energy change of the electron is most easily obtained by numerical integration of the electric field in Equation 10. For the experimental parameters in the LEAP experiment the laser beam waist size $w_{0}>>\lambda$ and hence the beam divergence $\left|\theta_{d}\right|<<1$. Furthermore 
the laser crossing angle is small $(\alpha<<1)$. Figure 4 shows the longitudinal electric field for a laser beam not terminated at the boundary. Notice that the region of interest where the laser field has a significant contribution is much smaller than the Rayleigh range $|z|<<z_{0}$. Furthermore notice that the amplitude of oscillation of the electric field has a gaussian-like envelope, which is mostly due to the finite transverse extent of the gaussian beam.

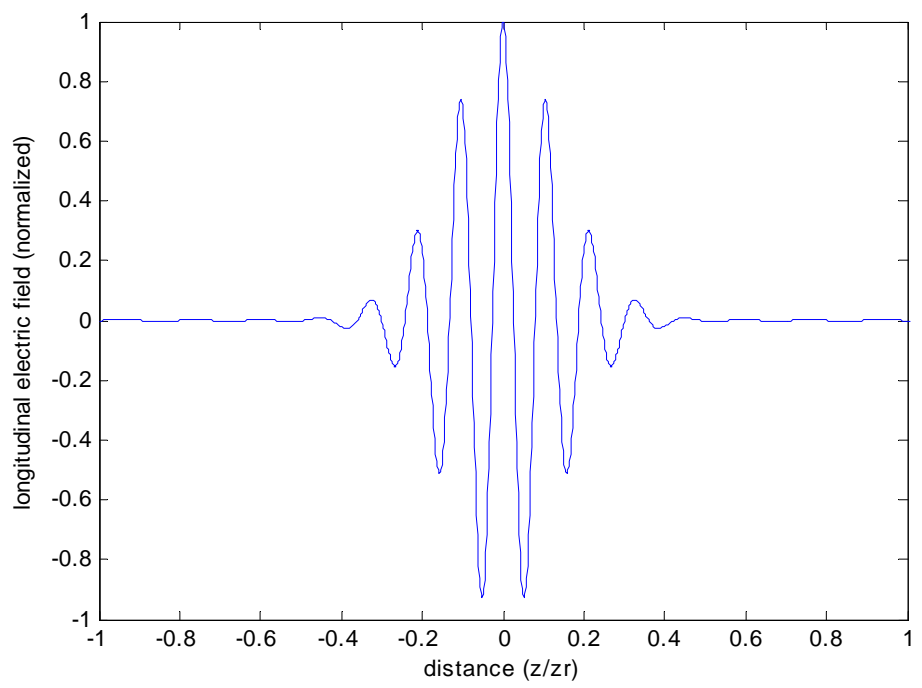

FIG 4: The longitudinal electric field for a single gaussian laser beam at a shallow angle to the electron beam

One can proceed and numerically integrate $E_{z}(z)$ to obtain the energy gain, however for the purpose of deriving an analytical expression assume that adjacent electric field oscillations nearly cancel except for the very last field oscillation in front or the terminating boundary.

To simplify Equation 8 assume the laser crossing angle $\alpha<<1$. Thus

$$
\psi_{t} \sim \omega t-k z\left(1-\alpha^{2}\right)+\eta(z)+\frac{k z^{2} \alpha^{2}}{2 R(z)}-\varphi
$$

Introducing the definitions of $\theta_{d}, z_{0}, w_{0}, \eta(z)$ and $R(z)$. The phase term of Equation 9 can be rewritten as

$$
\psi_{t} \sim \omega t-k z+\frac{\alpha^{2}}{\theta_{d}{ }^{2}} \hat{z}+\tan ^{-1} \hat{z}+\frac{\alpha^{2}}{\theta_{d}{ }^{2}\left(1+\hat{z}^{2}\right)} \hat{z}^{3}-\varphi
$$

since we assume that $|\hat{z}|<<1$ the inverse-tangent in Equation 10 can be approximated by $\tan ^{-1} x \sim x /\left(1+x^{2}\right)$ and hence the optical phase term simplifies further to 


$$
\psi_{t} \sim \omega t-k z+\frac{\alpha^{2}}{\theta_{d}^{2}} \hat{z}+\frac{\hat{z}}{1+\hat{z}^{2}}+\frac{\alpha^{2}}{\theta_{d}{ }^{2}\left(1+\hat{z}^{2}\right)} \hat{z}^{3}-\varphi
$$

The time variable can be eliminated by assuming the electron travels at a constant velocity, $z=v t$, or $t=z / v=z / c \beta$. With this in mind the optical phase term in Equation 11 becomes

$$
\psi_{t} \sim \hat{z}\left(\frac{1}{\gamma^{2} \theta_{d}^{2}}+\frac{\alpha^{2}}{\theta_{d}^{2}}\left(1+\theta_{d}^{2}+\frac{\hat{z}}{1+\hat{z}^{2}}\right)\right)-\varphi
$$

With the previously stated assumptions that $\omega_{0}>>\lambda$ and thus $\left|\theta_{d}\right|<<1$ the phase angle slips by $\pi$ in a distance much smaller that the Rayleigh range $z_{0}$. In this region where $|\hat{z}|<<1$ the phase term can be approximated by

$$
\psi_{t} \sim \frac{\hat{z}}{\theta_{d}^{2}}\left(\frac{1}{\gamma^{2}}+\alpha^{2}+\theta_{d}^{2}\right)-\varphi
$$

Notice that the optical phase term for crossed laser beams derived by Sprangle, Esarey and Krall contains $2 \theta_{d}{ }^{2}$ instead of $\theta_{d}{ }^{2}$. This difference is due to the inclusion of the intrinsic longitudinal electric field of a gaussian beam in their derivation that is neglected in this discussion, which is valid for the present situation where $\omega_{0}>\lambda$.

Since we are analyzing the limit where the gaussian beam is a near-plane wave $\theta_{d}{ }^{2}<<\alpha^{2}$. Hence this difference of $\theta_{d}{ }^{2}$ has no significance. The electric field in Equation 8 becomes

$$
E_{z} \sim E_{0} \alpha \cdot \cos \rho \cdot e^{-\left(\frac{\alpha}{\theta_{d}} \hat{z}\right)^{2}} \cos \psi_{t}
$$

With the assumption that the laser is a near-plane wave and that all except for the very last half electric field oscillation cancel the integral expression of Equation 1 becomes

$$
\Delta U=\int_{z}^{z+Z_{\text {slipage }} / 2} q \vec{E}(\vec{r}, t) d \vec{r}
$$

The slippage distance is given by the condition where the optical phase advances by $\pi$.

$$
\psi_{t}\left(Z_{\text {slippage }}\right)=\pi=\frac{Z_{\text {slippage }}}{Z_{0} \theta_{d}{ }^{2}}\left(\frac{1}{\gamma^{2}}+\alpha^{2}+\theta_{d}^{2}\right)
$$


Thus

$$
Z_{\text {slippage }}=\frac{\lambda}{\frac{1}{\gamma^{2}}+\alpha^{2}+\theta_{d}^{2}} \sim \frac{\lambda}{\frac{1}{\gamma^{2}}+\alpha^{2}}
$$

Since we are assuming that the amplitude factor has no appreciable change over $Z_{\text {slippage }}$, that is $\exp \left(\alpha^{2} \hat{z}^{2} / \theta_{d}{ }^{2}\right) \approx 1$ in the region of interest, the energy gain is approximately

$$
\Delta U \sim q E_{0} e^{-i \varphi} \cos \rho \cdot \alpha \int_{0}^{+Z_{\text {sippage }} / 2} \cos \left(\pi z / Z_{\text {slippage }}-\varphi\right) d z
$$

Thus the energy change for an electron estimated by the path integral method is

$$
\Delta U \sim \frac{\lambda q E_{0}}{\pi} \frac{\alpha}{\frac{1}{\gamma^{2}}+\alpha^{2}} \cos \rho \cdot \cos \varphi
$$

From Equation 19 we can see that the energy change depends on the optical phase $\varphi$, that it scales linearly with the electric field amplitude of the laser $E_{0}$, that it follows a cosine dependence with the polarization angle $\rho$, and that it scales linearly with the laser wavelength $\lambda$. Furthermore it shows a dependence with the laser crossing angle that scales as $f(\alpha) \propto \alpha /\left(1 / \gamma^{2}+\alpha^{2}\right)$ that has an optimum angle of $\alpha_{\max }=1 / \gamma$. Figure 5 shows that for the LEAP experiment laser and electron beam parameters the analytical approximation of Equation 19 is in good agreement with the numerical path integral of the longitudinal electric field of the laser beam.

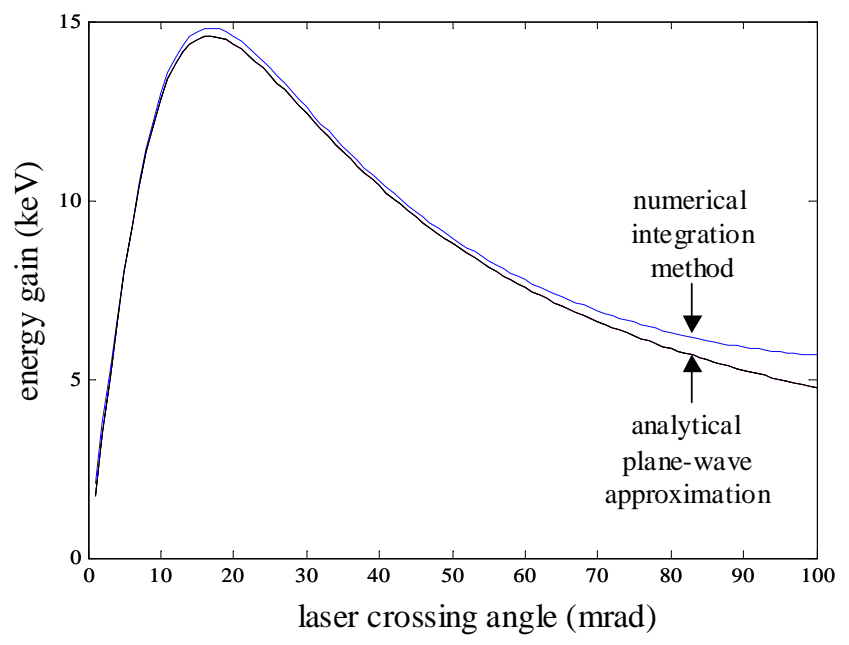

FIG 5: comparison between analytical approximation and numerical integral of the longitudinal electric field 


\section{THE INVERSE TRANSITION RADIATION PICTURE}

We proceed to calculate the electric retarded field of an electron approaching an infinite conductive boundary at normal incidence, and model it as a superposition of a charge and an image charge approaching each other at uniform velocity and stopping abruptly at the boundary. Figure 6 illustrates the situation, where the electric field at the observation point $\mathrm{O}$ with coordinates $(R, \theta, \phi)$ is to be calculated.

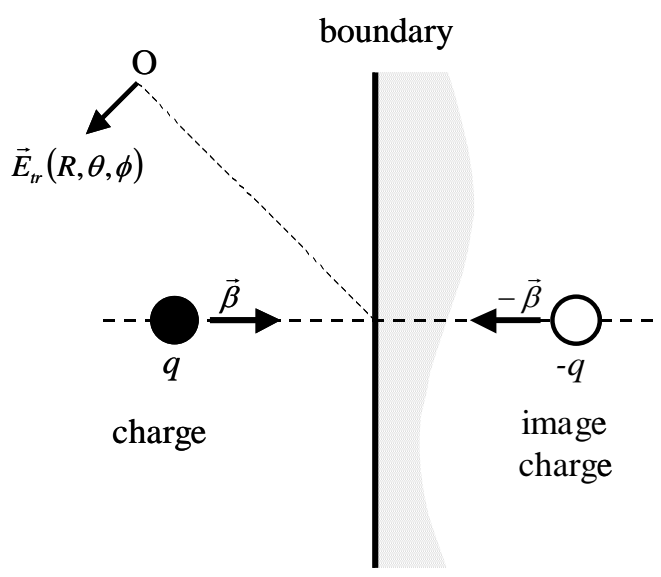

FIG 6: transition radiation calculation by the method of images

Starting from the retarded scalar and vector potentials for a point charge

$$
\begin{aligned}
& \vec{A}_{r e t}(\vec{x}, t)=\frac{\mu_{0} q}{4 \pi} \int_{-\infty}^{\infty} \frac{\vec{v} \delta\left(t^{\prime}-t+R / c\right)}{R\left(\vec{x}, t ; t^{\prime}\right)} d t^{\prime} \\
& \Phi_{r e t}(\vec{x}, t)=\frac{q}{4 \pi \varepsilon_{0}} \int_{V} \frac{\delta\left(t^{\prime}-t+R / c\right)}{R\left(\vec{x}, t ; t^{\prime}\right)} d t^{\prime}
\end{aligned}
$$

The retarded electric field of an accelerating charge can be found to be

$$
\vec{E}_{r a d}=\frac{q Z_{0}}{4 \pi K R} \frac{d}{d t^{\prime}}\left(\frac{\hat{n} \times(\hat{n} \times \vec{\beta})}{K}\right)
$$

In the frequency spectrum this corresponds to

$$
\begin{aligned}
& \vec{E}(\vec{x}, \omega)=\frac{q Z_{0}}{4 \pi R} \int_{-\infty}^{\infty} \hat{n} \times \frac{d}{d t^{\prime}}\left(\frac{\hat{n} \times \vec{\beta}}{K}\right) e^{-i \omega\left(t^{\prime}+R / c\right)} d t^{\prime}, \omega \geq 0 \\
& \vec{E}(\vec{x}, \omega)=0, \omega \leq 0
\end{aligned}
$$

so that in the time domain 


$$
E(t)=\frac{1}{2 \pi} \mathrm{RE}\left(\int_{-\infty}^{\infty} E(\omega) e^{i \omega t} d \omega\right)
$$

Assume the collision time is infinitely short, hence the term $e^{-i \omega\left(t^{\prime}+R / c\right)}$ is nearly constant over the integration time where $\beta$ changes value. Hence the total field caused by the charge plus the image charge is

$$
\vec{E}_{t r}(\vec{x}, \omega) \sim \frac{q Z_{0}}{4 \pi R} \hat{n} \times\left(\left.\frac{\hat{n} \times \vec{\beta}}{K}\right|_{\beta} ^{0}-\left.\frac{\hat{n} \times \vec{\beta}}{K}\right|_{-\beta} ^{0}\right) \sim \frac{q Z_{0}}{2 \pi R} \frac{\hat{n} \times(\hat{n} \times \vec{\beta})}{1-(\hat{n} \cdot \vec{\beta})^{2}}
$$

Note that the field is radially polarized

$$
\hat{n} \times(\hat{n} \times \vec{\beta})=\left(\begin{array}{c}
\cos \theta \cos \phi \\
\cos \theta \sin \phi \\
-\sin \theta
\end{array}\right)
$$

Hence in polar coordinates the transition radiation field is

$$
\vec{E}_{t r}(R, \theta, \phi, \omega)=\frac{q Z_{0}}{2 \pi R} \frac{\beta \sin \theta}{1-\beta^{2} \cos ^{2} \theta}\left(\begin{array}{c}
\cos \theta \cos \phi \\
\cos \theta \sin \phi \\
-\sin \theta
\end{array}\right)
$$

The compact expression of the electric field in radial coordinates implies that the overlap integral calculation between the laser field $\vec{E}_{\text {laser }}$ and the transition radiation field $\vec{E}_{t r}\left(\theta, \phi, \omega^{\prime}\right)$ is most conveniently carried out in the $(R \rightarrow \infty, \theta, \phi, \omega)$ coordinate system. Thus $\vec{E}_{\text {laser }}$ needs to be expressed as a superposition of plane waves in $(\theta, \phi, \omega)$ space. Written in phasor notation the scalar part of the laser field is

$$
E(\vec{r}, t)=\frac{E_{0} e^{-i \varphi}}{\sqrt{1+z^{\prime 2} / z_{0}^{2}}} e^{-\frac{x^{\prime 2}+y^{\prime 2}}{w\left(z^{\prime}\right)^{2}}} e^{i\left(\omega_{0} t-k z^{\prime}-\eta\left(z^{\prime}\right)-\frac{k x^{\prime 2}}{2 R\left(z^{\prime}\right)}\right)} \equiv A(\vec{r}) e^{i \omega_{0} t}
$$

Using the Fourier transformation pair that has the form 


$$
\begin{aligned}
& \tilde{E}(\vec{r}, \omega)=\int_{-\infty}^{\infty} E(r, t) e^{-i \omega t} d t \\
& E(r, t)=\frac{1}{2 \pi} \int_{-\infty}^{\infty} \tilde{E}(r, \omega) e^{i \omega t} d \omega
\end{aligned}
$$

Thus the spectrum of the (monochromatic) laser electric field is

$$
\widetilde{E}(\vec{r}, \omega)=2 \pi \delta\left(\omega-\omega_{0}\right) A(\vec{r})
$$

At $z=0$ the amplitude factor of the electric field is

$$
\tilde{A}(x, y, 0)=\left.E_{0} e^{-\frac{x^{\prime 2}+y^{\prime 2}}{w\left(z^{\prime}\right)^{2}}} e^{-i\left(\varphi+k z^{\prime}+\eta\left(z^{\prime}\right)+\frac{k x^{\prime 2}}{2 R\left(z^{\prime}\right)}\right)}\right|_{z=0}
$$

Assuming that the crossing angle $\alpha$ is small at the boundary $z=0$ the radius of curvature and Guoy phase shift terms can be neglected. Furthermore the laser spot size is $w\left(z^{\prime}\right) \sim w_{0}$. With these assumptions for $\tilde{A}(x, y, 0)$ the plane wave spectrum $\tilde{A}(u, v)$ for the amplitude becomes $\tilde{A}(x, y, 0)$ is (See appendix)

$$
\widetilde{A}(u, v)=\frac{1}{\lambda} \int_{-\infty}^{\infty} \int_{-\infty}^{\infty} E_{0} e^{-\frac{x^{2}+y^{2}}{w_{0}^{2}}} e^{-i k x u} e^{-i k y v} e^{-i\left(\varphi+k z^{\prime}\right)} d x d y
$$

Using the coordinate transformations in the small angle approximation $x^{\prime}=x \cos \alpha \sim x$, $y^{\prime}=y, z^{\prime}=-x \sin \alpha \sim-x \alpha$, and evaluating the integral in equation 31

$$
\tilde{A}(u, v)=\frac{\pi E_{0} w_{0}{ }^{2}}{\lambda} e^{-\frac{(u-\alpha)^{2}+v^{2}}{\theta_{d}^{2}}} e^{-i \varphi}
$$

To simplify the expression in the exponential term the definition of $\theta_{d}=\lambda / \pi w_{0}$ was used. Note that since $\theta_{d}<<1$ the exponential dies quickly. Assuming that the transition radiation field distribution has a negligible change over this the angular range the gaussian factor in Equation 32 can be approximated by a delta function

$$
e^{-\frac{(u-\alpha)^{2}+v^{2}}{\theta_{d}^{2}}} \sim \pi \theta_{d}^{2} \delta(u-\alpha, v)
$$

This is the equivalent assumption made in the path-integral calculation that the laser beam is a near-plane wave. Thus equation 32 simplifies further to 


$$
\tilde{A}(u, v)=E_{0} \lambda e^{-\frac{(u-\alpha)^{2}+v^{2}}{\theta_{d}^{2}}} e^{-i \varphi} \delta(u-\alpha, v)
$$

Note that the far-field paraxial angles $(u, v)$ form a rectangular coordinate system that expressed in the radial angle coordinates is $u=\sin \theta \cos \phi, v=\sin \theta \sin \phi$. Hence

$$
\tilde{A}(u, v)=\frac{E_{0} \lambda}{\sin \theta} e^{-\frac{(u-\alpha)^{2}+v^{2}}{\theta_{d}^{2}}} e^{-i \varphi} \delta(\theta-\alpha, \phi)
$$

The factor $1 / \sin \theta$ is a result of the coordinate system transformation. Adding the laser polarization vector for each of the two plane waves in Equation 35

$$
\left.\vec{E}_{\text {laser }}(\theta, \phi)\right|_{t} ^{o}=\frac{2 \pi E_{0} \lambda}{\sin \theta} e^{-\frac{(u-\alpha)^{2}+v^{2}}{\theta_{d}{ }^{2}}} e^{-i \varphi} \delta(\theta-\alpha, \phi) \delta\left(\omega-\omega_{0}\right)\left(\begin{array}{c}
\cos \theta \cos \rho \\
\sin \rho \\
-\sin \theta \cos \rho
\end{array}\right)
$$

This is the expression of the laser field traveling away from the focus in the far field and in the absence of a reflective screen. There is another term in $(\theta, \phi)$ coordinates for the incoming laser beam traveling into the focus. This incident field is located at the coordinates $(\theta=\pi-\alpha, \phi=\pi)$. Note that the vectors are written in Cartesian coordinates. Since the orientation of the vector of the incident laser beam is the same as the exiting beam the expression the $\mathrm{x}$-component picks up a negative sign when the radial coordinates undergo the transformation $(\theta=\alpha \rightarrow \theta=\pi-\alpha, \phi=0 \rightarrow \phi=\pi)$

$$
\left.\vec{E}_{\text {laser }}(\theta, \phi)\right|_{i}=\frac{2 \pi E_{0} \lambda}{\sin \theta} e^{-\frac{(u-\alpha)^{2}+v^{2}}{\theta_{d}{ }^{2}}} e^{-i \varphi} \delta(\theta-\alpha, \phi) \delta\left(\omega-\omega_{0}\right)\left(\begin{array}{c}
-\cos \theta \cos \rho \\
\sin \rho \\
-\sin \theta \cos \rho
\end{array}\right)
$$

In the presence of the boundary there are two more terms, one transmission term that is equal to $-\left.\vec{E}_{\text {laser }}(\theta, \phi)\right|_{t} ^{o}$ and that makes the total field behind the boundary zero, and a reflection term at coordinates $(\theta=\pi-\alpha, \phi=0)$.

$$
\left.\vec{E}_{\text {laser }}(\theta, \phi)\right|_{r}=-\frac{2 \pi E_{0} \lambda}{\sin \theta} e^{-\frac{(u-\alpha)^{2}+v^{2}}{\theta_{d}{ }^{2}}} e^{-i \varphi} \delta(\theta-\alpha, \phi) \delta\left(\omega-\omega_{0}\right)\left(\begin{array}{c}
-\cos \theta \cos \rho \\
\sin \rho \\
\sin \theta \cos \rho
\end{array}\right)
$$

Thus the total laser field in the presence of the boundary is

$$
\left.\vec{E}_{\text {laser }}(\theta, \phi)\right|_{s}=\left.\vec{E}_{\text {laser }}(\theta, \phi)\right|_{i}+\left.\vec{E}_{\text {laser }}(\theta, \phi)\right|_{r}
$$


Thus

$$
\begin{aligned}
\left.\vec{E}_{\text {laser }}(\theta, \phi)\right|_{s}= & \frac{2 \pi E_{0} e^{-i \varphi} \lambda}{\sin \theta} \delta\left(\omega-\omega_{0}\right) . \\
& \left\{\hat{n}_{1} \delta(\theta-(\pi-\alpha), \pi-\phi)-\hat{n}_{2} \delta(\theta-(\pi-\alpha), \phi)\right\}
\end{aligned}
$$

where

$$
\hat{n}_{1}=\left(\begin{array}{c}
-\cos \theta \cos \rho \\
\sin \rho \\
-\sin \theta \cos \rho
\end{array}\right), \hat{n}_{2}=\left(\begin{array}{c}
-\cos \theta \cos \rho \\
\sin \rho \\
\sin \theta \cos \rho
\end{array}\right)
$$

A diagram of the field components is shown in Figure 7

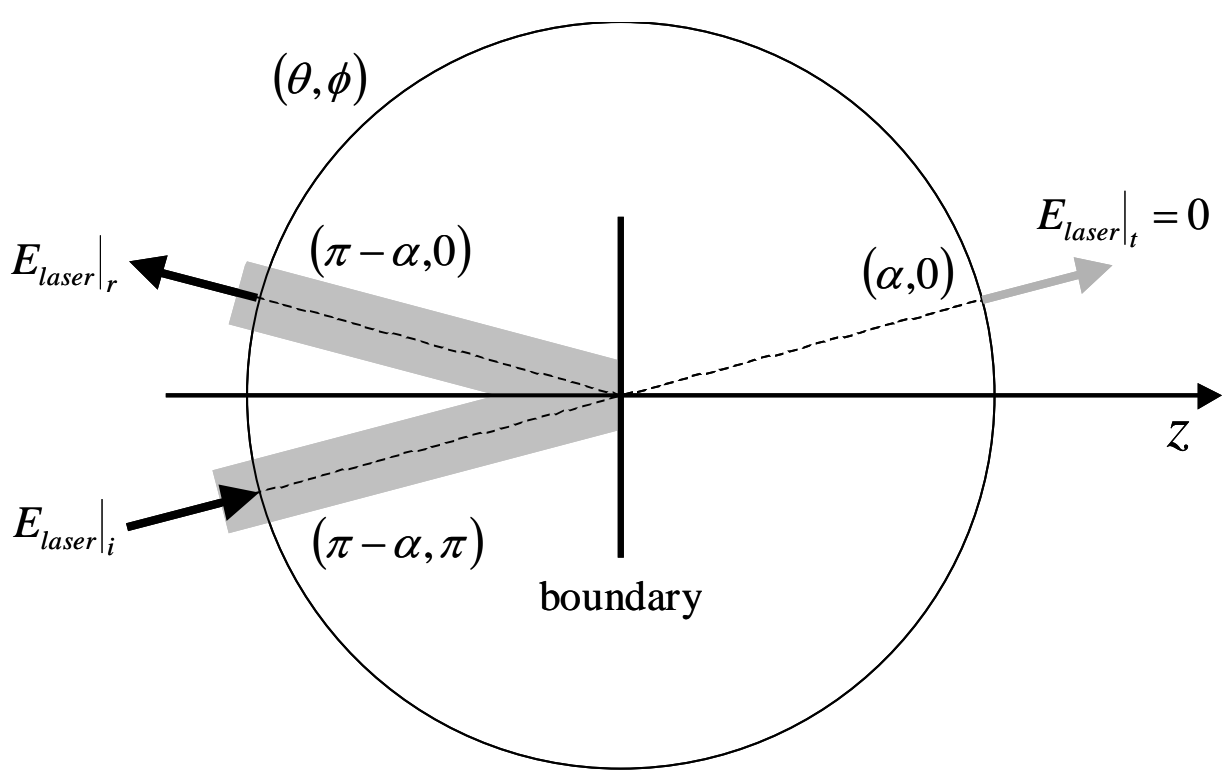

FIG 7: The laser field in the presence of the reflective boundary

Assuming no electromagnetic energy is stored in the volume of interaction and no ohmic losses Poyntings' Theorem predicts an energy change for the electron

$$
\Delta U=-\int_{-T}^{T} \oint \vec{S}(\theta, \phi, t) \cdot \hat{n} d a d t=-\frac{1}{Z_{0}} \int_{-T}^{T} \oint \vec{E}(\theta, \phi, t) \cdot \vec{E}(\theta, \phi, t) d a d t
$$

where it is assumed that $\vec{E}, \vec{B} \perp \hat{n}$ and $\vec{E} \perp \vec{B}$. This expression can be expanded to 


$$
\begin{aligned}
& \Delta U=-\int_{-T}^{T} \frac{1}{Z_{0}} \oint\left(\vec{E}_{\text {laser }}(t)+\vec{E}_{t r}(t)\right) \cdot\left(\vec{E}_{\text {laser }}(t)+\vec{E}_{t r}(t)\right) \cdot \hat{n} d \Omega d t \\
& \Delta U=-\int_{-T}^{T} \frac{1}{Z_{0}}\left(\oint \vec{E}_{\text {laser }}(t) \cdot \vec{E}_{\text {laser }}(t) d \Omega+\oint \vec{E}_{t r}(t) \cdot \vec{E}_{t r}(t) d \Omega+2 \oint \vec{E}_{\text {laser }}(t) \cdot \vec{E}_{t r}(t) d \Omega\right) d t
\end{aligned}
$$

Based on the assumption that boundary is a lossless high reflector

$$
\int_{-T \Omega}^{T} \oint_{\text {laser }}(\theta, \phi) \cdot \vec{E}_{\text {laser }}(\theta, \phi) d \Omega d t=0
$$

Furthermore following the assumption that the laser field magnitude is much larger than the transition radiation field magnitude we can neglect the transition radiation energy loss term $\vec{E}_{t r}(t) \cdot \vec{E}_{t r}(t)$ and are left with

$$
\Delta U \sim-\frac{2}{Z_{0}} \int_{-T}^{T} \oint \vec{E}_{\text {laser }}(t) \cdot \vec{E}_{t r}(t) d \Omega d t
$$

This is valid for real fields. For fields written as phasors the product of the fields in Equation 58 becomes the product of the real part of the phasors [12]

$$
\begin{aligned}
& \Delta U \sim-\frac{2}{Z_{0}} \int_{-T}^{T} \oint \operatorname{RE}\left(\widetilde{E}_{\text {laser }}(t)\right) \cdot \operatorname{RE}\left(\widetilde{E}_{t r}(t)\right) d \Omega d t \\
& =-\frac{1}{Z_{0}}\left(\int_{-T}^{T} \oint \operatorname{RE}\left(\tilde{E}_{\text {laser }}(t) \tilde{E}^{*}{ }_{t r}(t)\right) d \Omega d t+\int_{-T}^{T} \oint \operatorname{RE}\left(\widetilde{E}_{\text {laser }}(t) \tilde{E}_{\text {tr }}(t)\right) d \Omega d t\right)
\end{aligned}
$$

Changing the time dependent expression into a frequency dependent expression the time integral of Equation 46 becomes

$$
\begin{array}{r}
\int_{-\infty}^{\infty} E_{\text {laser }}(t) E_{t r}(t) d t=\frac{1}{2}\left(\frac{1}{2 \pi}\right)^{2}\left(\operatorname{RE} \int_{-\infty}^{\infty} \int_{-\infty}^{\infty} \int_{-\infty}^{\infty} E_{\text {laser }}(\omega) E_{t r}^{*}\left(\omega^{\prime}\right) e^{i \omega t} e^{-i \omega^{\prime} t} d \omega d \omega^{\prime} d t\right. \\
\left.+\operatorname{RE} \int_{-\infty}^{\infty} \int_{-\infty}^{\infty} \int_{-\infty}^{\infty} E_{\text {laser }}(\omega) E_{t r}\left(\omega^{\prime}\right) e^{i \omega t} e^{i \omega^{\prime} t} d \omega d \omega^{\prime} d t\right)
\end{array}
$$

which simplifies to

$$
\int_{-\infty}^{\infty} E_{\text {laser }}(t) E_{\text {tr }}(t) d t=\frac{1}{4 \pi} \operatorname{RE}\left(\int_{-\infty}^{\infty} E_{\text {laser }}(\omega) E_{\text {tr }}^{*}(\omega) d \omega+\int_{-\infty}^{\infty} E_{\text {laser }}(\omega) E_{t r}(-\omega) d \omega\right)
$$


Using the definitions of $E_{t r}$ in Equation 22 and of $E_{\text {laser }}$ in equation 29 the second integral in Equation 48 is zero and therefore

$$
\Delta U \sim-\frac{1}{2 \pi Z_{0}} \operatorname{RE}\left(\int_{-\infty}^{\infty} \oint \vec{E}_{\text {laser }}(\omega) \cdot \vec{E}^{*}{ }_{t r}(\omega) d \Omega d \omega\right)
$$

Inserting the expression for $\vec{E}_{\text {laser }}(\omega)$ and $\vec{E}_{\text {tr }}(\omega)$

$$
\begin{aligned}
\Delta U & \sim-\frac{q E_{0} \lambda}{4 \pi^{2}}\left(\int_{-\infty}^{\infty} 2 \pi \delta\left(\omega^{\prime}+\omega\right) d \omega\right) \operatorname{RE}\left(e^{-i \varphi}\right) \\
& \cdot \oint \frac{\left\{\hat{n}_{1} \delta(\theta-(\pi-\alpha), \pi-\phi)-\hat{n}_{2} \delta(\theta-(\pi-\alpha), \phi)\right\} \cdot \hat{n}_{t r} \beta \sin \theta}{\sin \theta\left(1-\beta^{2} \cos ^{2} \theta\right)} d \Omega
\end{aligned}
$$

The term $\operatorname{RE}\left(e^{-i \varphi}\right)$ is simply $\cos \varphi$. Integrating the delta function expressions and using the definitions of $\hat{n}_{1}, \hat{n}_{2}$ and $\hat{n}_{t r}$ the energy gain expression in Equation 50 simplifies to

$$
\Delta U \sim \frac{q E_{0} \lambda \cos \varphi}{\pi} \frac{\beta \sin \alpha}{1-\beta^{2} \cos ^{2} \alpha} \cos \rho
$$

Assuming $|\alpha|<<1$ and $\beta \sim 1$

$$
\Delta U \sim \frac{\lambda q E_{0} \cos \varphi}{\pi} \frac{\alpha}{\frac{1}{\gamma^{2}}+\alpha^{2}} \cos \rho
$$

One can conclude that for a laser beam terminated by a conductive boundary at normal incidence to the electron beam the energy gain expected from the path integral (Equation 19 ) is the same as the energy gain calculated by the inverse-radiation overlap integral method. 


\section{APPENDIX}

Define the plane wave decomposition of a field distribution $f(x, y)$ as

$$
\Psi(u, v)=C \int_{-\infty}^{\infty} \int_{-\infty}^{\infty} f(x, y) e^{-i k x u} e^{-i k y v} d x d y
$$

where $(u, v)$ are angular coordinates and $C$ is an arbitrary constant to be determined by conservation of total energy flux. The total energy flux of $f(x, y)$ has the form

$$
\Phi=\mathrm{P} \int_{-\infty}^{\infty} \int_{-\infty}^{\infty} f(x, y) f^{*}(x, y) d x d y
$$

The constant $\mathrm{C}$ is chosen such that

$$
\Phi=\mathrm{P} \int_{-\infty}^{\infty} \int_{-\infty}^{\infty} \Psi(u, v) \Psi^{*}(u, v) d u d v
$$

Thus from Equation A1

$$
\begin{aligned}
\Phi & =\mathrm{P} \int_{-\infty}^{\infty} \int_{-\infty}^{\infty} C \int_{-\infty}^{\infty} \int_{-\infty}^{\infty} f(x, y) e^{-i k x u} e^{-i k y v} d x d y C^{*} \int_{-\infty}^{\infty} \int_{-\infty}^{\infty} f^{*}\left(x^{\prime}, y^{\prime}\right) e^{+i k x^{\prime} u} e^{+i k y^{\prime} v} d x^{\prime} d y^{\prime} d u d v \\
& =\mathrm{PCC}^{*} \int_{-\infty}^{\infty} \int_{-\infty}^{\infty} \int_{-\infty}^{\infty} \int_{-\infty}^{\infty} \int_{-\infty}^{\infty} \int_{-\infty}^{\infty} f(x, y) f^{*}\left(x^{\prime}, y^{\prime}\right) e^{-i k x u} e^{-i k y v} e^{+i k x^{\prime} u} e^{+i k y^{\prime} v} d x^{\prime} d y^{\prime} d x d y d u d v \\
& =\mathrm{PCC}^{*} \int_{-\infty}^{\infty} \int_{-\infty}^{\infty} \int_{-\infty}^{\infty} \int_{-\infty}^{\infty} f(x, y) f^{*}\left(x^{\prime}, y^{\prime}\right) \int_{-\infty}^{\infty} \int_{-\infty}^{\infty} e^{-i k\left(x-x^{\prime}\right) u} e^{-i k\left(y-y^{\prime}\right) v} d u d v d x^{\prime} d y^{\prime} d x d y \\
& =\mathrm{PCC}^{*} \int_{-\infty}^{\infty} \int_{-\infty}^{\infty} \int_{-\infty}^{\infty} \int_{-\infty}^{\infty} f(x, y) f^{*}\left(x^{\prime}, y^{\prime}\right) \int_{-\infty}^{\infty} \int_{-\infty}^{\infty} e^{-i k\left(x-x^{\prime}\right) u} e^{-i k\left(y-y^{\prime}\right) v} d u d v d x^{\prime} d y^{\prime} d x d y \\
& =\mathrm{PCC}^{*} \int_{-\infty}^{\infty} \int_{-\infty}^{\infty} \int_{-\infty}^{\infty} \int_{-\infty}^{\infty} f(x, y) f^{*}\left(x^{\prime}, y^{\prime}\right)\left(\frac{2 \pi}{k}\right)^{2} \delta\left(x-x^{\prime}\right) \delta\left(y-y^{\prime}\right) d x^{\prime} d y^{\prime} d x d y \\
& =\mathrm{PCC}^{*} \lambda^{2} \int_{-\infty}^{\infty} \int_{-\infty}^{\infty} f(x, y) f^{*}(x, y) d x d y
\end{aligned}
$$

Thus $|C|=1 / \lambda$. Hence the plane wave decomposition is

$$
\Psi(u, v)=\frac{1}{\lambda} \int_{-\infty}^{\infty} \int_{-\infty}^{\infty} f(x, y) e^{-i k x u} e^{-i k y v} d x d y
$$




\section{REFERENCES}

1. T. Plettner et al, to appear in Phys. Rev. Lett. (2005)

2. R.H. Pantell, M.A. Piestrup, "Free-electron momentum modulation by means of limited interaction length with light”, Applied Physics Letters, 32, no. 11, p 781783 (1978)

3. E. Esarey, P. Sprangle, J. Krall, "Laser Acceleration of Electrons in Vacuum”, Physical Review E, 52, p 5443-5453 (1995)

4. E.J. Bochove, G.T. Moore, M.O. Scully, "Acceleration of particles by an asymmetric Hermite-Gaussian laser beam”, Physical Review A, Vol. 46 No. 10 p. 6640-6653 (1992)

5. J.A. Edinghofer, R.H. Pantell, "Energy exchange between free electrons and light in vacuum”, Journal of Applied Physics, 50, p 6120-6122 (1979)

6. W. D. Kimura, G. H. Kim, R. D. Romea, L. C. Steinhauer, I. V. Pogorelsky, K. P. Kusche, R. C. Fernow, X. Wang, Y. Liu, "Laser Acceleration of Relativistic Electrons Using the Inverse Cherenkov Effect”, Phys. Rev. Lett. 74, 546-549 (1995)

7. X.E. Lin, "Photonic band gap fiber accelerator”, Phys. Rev. ST Accel. Beams 4, 051301 (2001)

8. See for example Jackson, Classical Electrodynamics, $2^{\text {nd }}$ edition, chapter $6, \mathrm{p} 236$

9. M. Xie, Proceedings of the 2003 Particle Accelerator Conference (2003)

10. Z. Huang, G. Stupakov and M. Zolotorev, "Calculation and Optimization of Laser Acceleration in Vacuum”, Phys. Rev. Special Topics - Accelerators and Beams,Vol. 7, 011302 (2004)

11. A. Yariv, Optical Electronics, $4^{\text {th }}$ edition, Saunders College Publishing, p. 48

12. D.K. Cheng, Field and Wave Electromagnetics, $2^{\text {nd }}$ edition, Addison-Wesley Publishing Company, p. 383 